\title{
Optimal Designs for Stated Choice Experiments Generated from Fractional Factorial Designs
}

\author{
Stephen Bush \\ School of Mathematical Sciences, University of Technology Sydney, \\ PO Box 123 Broadway NSW 2007, Australia \\ Email: stephen.bush@uts.edu.au
}

\begin{abstract}
This paper gives optimal designs obtained by developing a fractional factorial design for the estimation of main effects in stated choice experiments under the assumption of equal selection probabilities. This construction approach follows that of Burgess and Street (2005), who develop complete factorial designs to construct optimal designs for choice experiments, but we obtain choice experiments with fewer choice sets. We construct the fractional factorial designs using the Rao-Hamming method, which assumes all attributes have the same number of levels, which must be a prime or a prime power. We also find optimal designs for stated choice experiments that are generated from asymmetric fractional factorial designs constructed using expansive replacement under the same assumption. We use the multinomial logit model to analyse the results, and we make the assumption of equal selection probabilities when calculating optimality properties. The methods that we use to implement these constructions are given in the last section.
\end{abstract}

AMS Subject Classification: 62K05, 05B15

Keywords: Multiple comparisons, Bradley-Terry model, Multinomial logit model, RaoHamming Construction, Expansive Replacement

\section{Introduction}

Stated choice experiments, or discrete choice experiments (DCEs) are becoming an increasingly popular method for eliciting preferences across many different disciplines. For example, Lancsar et al. (2007) use DCEs to determine the most important outcomes for preventative asthma treatments, Jaeger et al. (2008) use DCEs to investigate consumer preferences for fruit purchases, and Carson et al. (2009) use DCEs to gauge public support for climate change policy in Australia. A comprehensive introduction to the area can be found in Louviere et al. (2000), and in Train (2003).

In a DCE, we present a series of choice sets, in turn, to each respondent. Each choice set consists of a number of alternatives from which respondents are asked to choose the 
option that they think is best. We assume that each respondent is shown the same set of choice sets, and that all choice sets contain the same number of alternatives.

In this paper, we assume that the multinomial logit model (MNL model) is used to estimate the effect of each attribute on the probability that an alternative is selected. The appeal of this model is that the likelihood function has a closed form, which means that it is not necessary to use simulation methods to obtain parameter estimates. While the limitations of this model, such as independence from irrelevant alternatives, are widely discussed in the literature (Louviere et al. (2000), Train (2003)), the designs that are efficient for the estimation of the MNL model have been shown to perform well for other models (Burgess et al. (2011)). In choice sets of size 2, and after a suitable reparameterisation, the MNL model coincides with the Bradley-Terry model (Bradley and Terry (1952)).

One important step in conducting a DCE is determining which sets of alternatives are selected to form choice sets, and which set of choice sets is used for the DCE. That is, how do we design the DCE? This question is important because a poor design will require more choice sets than an efficient design to achieve the same level of precision in the parameter estimates.

There are many criteria that can be used to compare designs. Several of these are based on the asymptotic properties of the Fisher information matrix (or simply the information matrix) for the model that is to be fitted. In this paper we use the $D$-optimality criterion, which finds the design that maximises the determinant of the information matrix.

Unlike the case for linear models, the information matrix for nonlinear models, such as the MNL model, is a function of the parameters that are to be estimated, which are unknown when we choose a design. To overcome this problem, we assume that all alternatives are equally attractive to the respondent, and use this assumption to find locally optimal designs. That is, we assume that each alternative has an equal probability of selection. This reduces the problem to the corresponding problem in a linear model. An alternative approach is to specify a prior distribution and find a Bayesian optimal design; see Kessels et al. (2009) for a discussion of this approach.

Burgess and Street (2003) prove a theorem that provides $D$-optimal designs for the estimation of main effects, where all attributes have two levels, and the design has been developed from a complete factorial design. In Street and Burgess (2004), the authors reduce the number of choice sets required to obtain a $D$-optimal design by proving a theorem that gives $D$-optimal designs for the estimation of main effects, where all attributes have two levels, and the design has been developed from a fractional factorial design. Burgess and Street (2005) prove a theorem that provides $D$-optimal designs for the estimation of main effects, where attributes may take any number of levels, and the design has been developed from a complete factorial design. While Chapter 7 of Street and Burgess (2007) provides constructions that develop fractional factorial designs to obtain a choice design with attributes that are not restricted to two levels, they do not prove that such designs can give $D$-optimal designs under certain conditions.

The purpose of this paper, and indeed the novel contribution of this paper, is to present and prove theorems that give $D$-optimal designs that are developed from fractional factorial designs when attributes have more than two levels. In Section 3, we present a theorem that gives $D$-optimal designs for the estimation of main effects where 
all attributes have the same number of levels and the number of levels is a prime or a prime power, and the choice design is developed from a fractional factorial design. In Section 4 , we present a theorem that gives $D$-optimal designs for the estimation of main effects where the attributes may take different numbers of levels, but all powers of the same prime, and the choice design is developed from a fractional factorial design. In Section 5 we provide some advice about how to choose appropriate sets of generators to develop the fractional factorial design such that the conditions of the theorems in Burgess and Street (2005), as well as Theorems 3.1 and 4.1 of this paper are satisfied.

The supplementary material for this paper provides full derivations of the information matrices, as well as proofs of Theorems 3.1 and 4.1. This supplementary material is available at http://sites.google.com/site/stephenabush/.

\section{Preliminary Results and Notation}

In this section, we review some concepts that will be useful when constructing optimal designs for the MNL model. We introduce relevant notation, review some properties of the MNL model and results of Burgess and Street (2005), which we extend in this paper, using the Rao-Hamming construction method and the expansive replacement method.

We begin with some notation. We say that a choice experiment consists of $N$ choice sets, each containing $m$ alternatives (also known as profiles, options, or items), from which the respondent is asked to choose the most preferred. We describe each alternative by a set of $k$ attributes. We assume that the $q^{\text {th }}$ attribute has $\ell_{q}$ levels, represented by $0,1, \ldots, \ell_{q}-1$. Thus we describe each alternative as a $k$-tuple of attribute levels. We also assume that no choice set contains a repeated alternative.

Once we obtain responses from each of the $s$ respondents, we use the MNL model to model respondent preferences. In the MNL model we assign a value $\pi_{i}$ to each alternative $T_{i}$, where $i=1, \ldots, L$ and $L=\prod_{q=1}^{k} \ell_{q}$, which represents the attractiveness of the alternative to the respondent. The larger the value of $\pi_{i}$, the more attractive the alternative $T_{i}$ is to the respondent. Then, for a choice set $C=\left\{T_{i_{1}}, T_{i_{2}}, \ldots, T_{i_{m}}\right\}$, the probability that an alternative $T_{i} \in C$ is selected from the choice set is $P\left(T_{i} \mid C\right)=$ $\pi_{i} /\left(\sum_{a=1}^{m} \pi_{i_{a}}\right)$, with the normalising constraint $\prod_{i=1}^{L} \pi_{i}=1$. In this case, Burgess and Street (2003) derive the Fisher information matrix for the estimation of $\sqrt{s N} \gamma$, where $\gamma=\left(\gamma_{1}, \ldots, \gamma_{L}\right)^{T}$ and we assume that $\gamma_{i}=\ln \left(\pi_{i}\right)$ is a linear combination of attribute effects for alternative $T_{i}$. We introduce these linear combinations in the form of contrast matrices later. The authors also let $\lambda_{C}$ be the proportion of how often the choice set $C$ appears in the experiment. The authors show that the $i^{\text {th }}$ diagonal entry of the $L \times L$ information matrix $\Lambda(\boldsymbol{\pi})$ is given by

$$
\pi_{i} \sum_{C \mid T_{i} \in C} \frac{\lambda_{C}\left(\sum_{a=1}^{m} \pi_{i_{a}}-\pi_{i}\right)}{\left(\sum_{b=1}^{m} \pi_{i_{b}}\right)^{2}},
$$

and the $(i, j)^{\text {th }}$ entry of the information matrix $\Lambda(\pi)$, where $i \neq j$, is given by

$$
-\pi_{i} \pi_{j} \sum_{C \mid T_{i}, T_{j} \in C} \frac{\lambda_{C}}{\left(\sum_{b=1}^{m} \pi_{i_{b}}\right)^{2}},
$$


Table 2.1: The structure of a design constructed from $F$ using generators

\begin{tabular}{cccc}
\hline Option 1 & Option 2 & $\ldots$ & Option m \\
\hline$F$ & $F+\mathbf{g}_{1,2}$ & $\ldots$ & $F+\mathbf{g}_{1, m}$ \\
$F$ & $F+\mathbf{g}_{2,2}$ & $\ldots$ & $F+\mathbf{g}_{2, m}$ \\
$\vdots$ & $\vdots$ & & $\vdots$ \\
$F$ & $F+\mathbf{g}_{\zeta, 2}$ & $\ldots$ & $F+\mathbf{g}_{\zeta, m}$ \\
\hline
\end{tabular}

where $\boldsymbol{\pi}=\left(\pi_{1}, \ldots, \pi_{L}\right)^{T}$, and the summation is over all of the distinct choice sets. Under the assumption of equal selection probabilities, that is $P\left(T_{i_{a}} \mid C\right)=1 / m$ for $1 \leq a \leq m$, the $i^{\text {th }}$ diagonal entry becomes $\sum_{C \mid T_{i} \in C}(m-1) \lambda_{C} / m^{2}$, and the $(i, j)^{\text {th }}$ entry, where $i \neq j$, becomes $-\sum_{C \mid T_{i}, T_{j} \in C} \lambda_{C} / m^{2}$. This assumption is equivalent to assuming that $\gamma_{i}=0$ for $1 \leq i \leq L$.

In DCEs, we are not particularly interested in estimating the $\pi_{i}$ values for each alternative, rather we are interested in estimating the main effects of the attributes, or interaction effects between attributes. In this paper, we focus on main effects only. With this in mind, we need to reparameterise the Fisher information matrix so that we are estimating the contrasts of the $\gamma_{i}$ that correspond to the attribute main effects. So, following El-Helbawy and Bradley (1978), we define a matrix $B$, with dimensions $p \times L$ and orthonormal rows, that contains the coefficients of the $p=\sum_{q=1}^{k}\left(\ell_{q}-1\right)$ contrasts that correspond to the main effects that are to be estimated for each of the $k$ attributes. Then $C(\boldsymbol{\pi})=B \Lambda(\boldsymbol{\pi}) B^{T}$ is the Fisher information matrix for the estimation of $\sqrt{s N} B \boldsymbol{\gamma}$. A $D$-optimal design will then be a design that maximises $\operatorname{det}(C(\boldsymbol{\pi}))$. $D$-optimal designs are not necessarily unique.

There are several results in the literature that give optimal, or near-optimal, designs for DCEs in various circumstances. The designs we consider in this paper are constructed from a starting design, to which we add generators. The starting design, $F$, is either a complete factorial design or a fractional factorial design, whose entries become the first alternatives in each choice set. Here, we use the term starting design in the same way as Chapter 8 of Street and Burgess (2007), which should not be confused with the starting designs that are used in search algorithms. We then add $m-1$ generators, in turn, component-wise modulo $\ell_{q}$, to each row of the starting design. These form the remaining alternatives in each choice set. Since position effects are not of interest in this paper, the order of the alternatives within each choice set is fixed, but arbitrary; so the alternatives in $F$ may, in fact, be placed in any of the positions of the choice set as long as they are all in the same position (so all of the alternatives in $F$ may appear in the second position of the choice set, for instance). In general, a choice set is generated using $\zeta$ sets of $m$ generators $G_{\alpha}=\left(\boldsymbol{g}_{\alpha, 1}=\mathbf{0}, \boldsymbol{g}_{\alpha, 2}, \ldots, \boldsymbol{g}_{\alpha, m}\right)$, for $\alpha=1,2, \ldots, \zeta$, and where $\boldsymbol{g}_{\alpha, i}=\left(g_{\alpha, i, 1}, g_{\alpha, i, 2}, \ldots, g_{\alpha, i, k}\right)$, for $i=1,2, \ldots, m$. To ensure that there are no repeated alternatives within a choice set, we make the restriction $\boldsymbol{g}_{\alpha, 2} \neq \mathbf{0}, \ldots, \boldsymbol{g}_{\alpha, m} \neq \mathbf{0}$. The structure of the resulting design is shown in Table 2.1. The following example illustrates this construction.

Example 2.1. Suppose that we have an experiment with $m=3, \zeta=1$, and $\ell_{1}=\ell_{2}=$ 
Table 2.2: Choice Design with $k=4, m=3$ and $\ell_{q}=3$

\begin{tabular}{cccccccccccc}
\hline \multicolumn{3}{c}{ Option 1 } & \multicolumn{4}{c}{ Option 2 } & \multicolumn{3}{c}{ Option 3 } \\
\hline 0 & 0 & 0 & 0 & 1 & 2 & 1 & 2 & 2 & 1 & 2 & 1 \\
0 & 1 & 1 & 1 & 1 & 0 & 2 & 0 & 2 & 2 & 0 & 2 \\
0 & 2 & 2 & 2 & 1 & 1 & 0 & 1 & 2 & 0 & 1 & 0 \\
1 & 0 & 1 & 2 & 2 & 2 & 2 & 1 & 0 & 1 & 0 & 0 \\
1 & 1 & 2 & 0 & 2 & 0 & 0 & 2 & 0 & 2 & 1 & 1 \\
1 & 2 & 0 & 1 & 2 & 1 & 1 & 0 & 0 & 0 & 2 & 2 \\
2 & 0 & 2 & 1 & 0 & 2 & 0 & 0 & 1 & 1 & 1 & 2 \\
2 & 1 & 0 & 2 & 0 & 0 & 1 & 1 & 1 & 2 & 2 & 0 \\
2 & 2 & 1 & 0 & 0 & 1 & 2 & 2 & 1 & 0 & 0 & 1 \\
\hline
\end{tabular}

$\ell_{3}=\ell_{4}=3$. To construct a design for this experiment, we add two generators to a fractional factorial design $F$, which is given in the first block of columns in Table 2.2. Let the first of these generators be $\boldsymbol{g}_{1,2}=(1212)$. Then by adding $\boldsymbol{g}_{1,2}$ to each row of $F$ component-wise modulo 3 , we obtain the options given in the second set of columns in Table 2.2. Similarly, we add $\boldsymbol{g}_{1,3}=(2121)$ component-wise modulo 3 to each row of $F$ to obtain the final option of each choice set. Then each row describes a choice set of size 3 .

We can characterise a set of generators by looking at the differences between attribute levels for pairs of options. To do this, we construct a multiset of differences between ordered pairs of generators for particular attributes. A multiset is defined not only by the elements contained in the set, but also by the frequency with which each element appears in the set. So if we consider the first attribute in Example 2.1, the multiset of differences between each ordered pair is $\left\{g_{1,1,1}-g_{1,2,1}=2, g_{1,1,1}-g_{1,3,1}=\right.$ $\left.1, g_{1,2,1}-g_{1,1,1}=1, g_{1,2,1}-g_{1,3,1}=2, g_{1,3,1}-g_{1,1,1}=2, g_{1,3,1}-g_{1,2,1}=1\right\}$, since $\boldsymbol{g}_{1,1}=(0000)$. So this multiset contains no $0 \mathrm{~s}$, three 1s and three 2s. In Theorem 2.1, we place conditions on these multisets that give optimal designs.

In this paper, we build on the following theorem, proven in Burgess and Street (2005), that gives optimal designs for the estimation of main effects when the starting design is a complete factorial design and $\zeta=1$.

Theorem 2.1. (Burgess and Street (2005)) Let $F$ be the complete factorial for $k$ attributes where the $q^{\text {th }}$ attribute has $l_{q}$ levels. Suppose that we choose a set of $m$ generators $G=\left\{\boldsymbol{g}_{1}=\mathbf{0}, \boldsymbol{g}_{2}, \ldots, \boldsymbol{g}_{m}\right\}$ such that $\boldsymbol{g}_{i} \neq \boldsymbol{g}_{j}$ for $i \neq j$. Suppose that $\boldsymbol{g}_{i}=$ $\left(g_{i, 1}, g_{i, 2}, \ldots, g_{i, k}\right)$ for $i=1, \ldots m$ and suppose that the multiset of differences for attribute $q, \uplus_{i \neq j}\left(g_{j, q}-g_{i, q}\right)$, contains each non-zero difference modulo $\ell_{q}$ equally often. Then the choice sets given by the rows of $F+\boldsymbol{g}_{1}, F+\boldsymbol{g}_{2}, \ldots, F+\boldsymbol{g}_{m}$, for one or more sets of generators $G$, are optimal for the estimation of main effects only, provided that there are as few zero differences as possible in each choice set.

To obtain optimal designs using this theorem we require two things. The first is a complete factorial design, and the second is a set of $m$ generators that satisfy the conditions stated in Theorem 2.1. If $m=\ell$, the number of levels for each attribute, 
we can select entries for the set of generators such that each of the non-zero values, $1, \ldots, \ell-1$, appears in the $q^{\text {th }}$ position of exactly one of the $m-1$ generators. The generators in Example 2.1 satisfy this condition. If $m \neq \ell$, this problem becomes more difficult, and more than one set of generators may be necessary to satisfy this condition. This difficulty is discussed in Section 5 of Street and Burgess (2008). If we are using more than one set of generators then we require the multiset sum of the multisets of differences for each set of generators to contain each non-zero difference equally often. We discuss the existence of appropriate sets of generators further in Section 5 .

In Theorem 2.1, the class of competing designs is restricted so that "any experiment contains all choice sets with a particular difference vector equally often". In this context, we define the difference vector for a choice set constructed from the $\alpha^{\text {th }}$ set of generators to contain $m(m-1) / 2$ entries, corresponding to the $m(m-1) / 2$ pairs of positions in the choice set, where each entry is a $k$-tuple of 0 s and $1 \mathrm{~s}$. For the $q^{\text {th }}$ position in the $k$-tuple corresponding to the pair of alternatives in positions $i$ and $j$ of the choice set, the corresponding entry in the difference vector equals 0 if $g_{\alpha, j, q}-g_{\alpha, i, q}=0$, and equals 1 otherwise.

Example 2.2. Consider the design in Table 2.2. In this experiment, $\boldsymbol{g}_{1,2}-\boldsymbol{g}_{1,1}=(1212)$, $\boldsymbol{g}_{1,3}-\boldsymbol{g}_{1,1}=(2121)$, and $\boldsymbol{g}_{1,3}-\boldsymbol{g}_{1,2}=(1212)$. So the difference vector for each choice set in this design is $\{(1111),(1111),(1111)\}$, since each entry in the three differences is non-zero. If we have $\boldsymbol{g}_{1,3}=(2222)$ instead, then $\boldsymbol{g}_{1,2}-\boldsymbol{g}_{1,1}=(1212), \boldsymbol{g}_{1,3}-\boldsymbol{g}_{1,1}=(2222)$, and $\boldsymbol{g}_{1,3}-\boldsymbol{g}_{1,2}=(1010)$. The difference vector will then be $\{(1111),(1111),(1010)\}$.

In this paper, the class of competing designs are all designs that can be constructed by adding one or more sets of generators to an orthogonal array constructed using Constructions 2.1 and 2.2, where generators satisfy the condition that the multiset of differences for each attribute $q$ over all sets in the collection $\uplus_{\alpha=1}^{\zeta} \uplus_{i \neq j}\left(g_{\alpha, j, q}-g_{\alpha, i, q}\right)$ contains each non-zero difference in the Galois field of order $\ell_{q}, G F\left[\ell_{q}\right]$, equally often. In the condition of the multiset of differences, we only count the differences for each attribute within each set of generators.

One problem with the optimal designs arising from Theorem 2.1 is that the number of choice sets becomes large quite quickly as the number of attributes increases, or as the number of levels that each attribute may take increases (Großmann et al. (2007), Street and Burgess (2007) Chapter 8). Since researchers need to balance design optimality with respondent burden, small, optimal or near-optimal designs are useful in practice.

Graßhoff et al. (2004) provides several constructions for the case where $m=2$, and proves that the resulting designs are optimal. In particular, the authors use Hadamard matrices and orthogonal arrays to create a design structure into which pairs of levels are embedded. For symmetric experiments, that is when $\ell_{1}=\ldots=\ell_{k}=\ell$, the authors also use Bhaskar-Rao designs to find optimal designs with $\ell(\ell-1) / 2$ choice sets. Graßhoff and Schwabe (2008) prove results that give optimal designs when $k=1$ or $2, m=2$, without making the assumption of equal selection probabilities.

Street et al. (2005) also investigate different methods of obtaining small nearoptimal designs for choice experiments with two or more alternatives in each choice set. The authors find that by using a fractional factorial starting design they consistently obtain efficient designs that allow for the independent estimation of main effects, or main effects plus two-factor interactions when all attributes may take two levels. The authors present upper bounds for the determinant of the information matrix, but do 
not present any general results about which designs may be optimal when a fractional factorial starting design is used.

In this paper, we use fractional factorial designs that are specified by orthogonal arrays to obtain the starting design $F$. Street and Burgess (2007) define an orthogonal array $O A\left[N ; \ell_{1}, \ell_{2}, \ldots, \ell_{k} ; t\right]$ of strength $t$ to be an $N \times k$ array with the elements in column $q$ chosen from a set of $\ell_{q}$ symbols such that any $N \times t$ subarray has each $t-$ tuple appearing as a row an equal number of times. The orthogonal array is said to be symmetric if $\ell_{1}=\ell_{2}=\ldots=\ell_{k}$, and is said to be asymmetric otherwise. We denote a symmetric orthogonal array with $N$ runs, $k$ columns, and of strength $t$ with a common number of levels $\ell$ by $O A[N, k, \ell, t]$. Since we are considering fractional factorial designs specified by orthogonal arrays, as discussed in Section 11.4 of Hedayat et al. (1999), we will refer to the starting designs as orthogonal arrays.

Rao $(1947,1949)$ provides a method for constructing an $O A\left[\ell^{n},\left(\ell^{n}-1\right) /(\ell-1), \ell, 2\right]$ where the number of levels for each attribute is a prime or a prime power (Hedayat et al. (1999), p. 49). This construction method uses the properties of Galois fields of order $\ell, G F[\ell]$, which exist when $\ell$ is a prime or a prime power. Appendix A of Hedayat et al. (1999) provides a good introduction to Galois fields. Since primes are special cases of prime powers we will only refer to prime powers. This construction is known as the RaoHamming construction, since the same construction is also used to obtain the Hamming codes introduced in Hamming (1950).

Construction 2.1. (from Hedayat et al. (1999), p. 50) Form an $\ell^{n} \times n$ array with all possible $n$-tuples from $G F[\ell]$, where $\ell$ is a prime power. Let $C_{1}, \ldots, C_{n}$ denote the columns of this array. The columns of the $O A\left[\ell^{n},\left(\ell^{n}-1\right) /(\ell-1), \ell, 2\right]$ then consist of all columns of the form

$$
a_{q, 1} C_{1}+a_{q, 2} C_{2}+\ldots+a_{q, n} C_{n}=\left[C_{1}, C_{2}, \ldots, C_{n}\right] \boldsymbol{a}_{q},
$$

where $\boldsymbol{a}_{q}=\left(a_{q, 1}, \ldots, a_{q, n}\right)^{T}$ is an $n$-tuple from $G F[\ell]$, not all the $a_{q, i}$ are zero, and the first non-zero $a_{q, i}$ is 1 .

A comprehensive catalogue of orthogonal arrays is provided on Neil Sloane's website http://www2.research.att.com/ njas/oadir/index.html.

In this paper, we use orthogonal arrays to reduce the number of choice sets arising from the construction in Theorem 2.1. In this approach, an orthogonal array is used as the starting design $F$, rather than the complete factorial design. Such an approach was considered in Section 8.1 of Street and Burgess (2007), however the authors considered near-optimal designs rather than optimal designs, as we do here. Initially we will consider symmetric designs, that is $\ell_{1}=\ldots=\ell_{k}=\ell$, where $\ell$ is a prime power. In Section 4 , we look at some asymmetric designs where the orthogonal array has been obtained through the expansive replacement of columns of a Rao-Hamming design. Expansive replacement (Hedayat et al. (1999), Chapter 9) involves replacing the elements in a column of an $O A\left[N, k_{1}, \ell_{1}, 2\right]$ with the rows of a second OA, $O A\left[\ell_{1}, k_{2}, \ell_{2}, 2\right]$.

Construction 2.2. Suppose that $\ell_{1}=s^{q_{1}}$, that $\ell_{2}=s^{q_{2}}$, where $s$ is a prime and $q_{2}<q_{1}$, and that there exist two fractional factorial designs $F_{1}$ and $F_{2}$, where $F_{1}$ is an $\ell_{1}^{k_{1}}$ design with $N$ runs and $F_{2}$ is an $\ell_{2}^{k_{2}}$ design with $\ell_{1}$ runs. Then in expansive replacement, we replace the $r^{\text {th }}$ attribute of the first design by $k_{2}$ attributes, each with $\ell_{2}$ levels, by making 
Table 2.3: Choice Design with $k=7, m=2$ and $\ell_{q}=2$

\begin{tabular}{lllllllllllllll}
\hline \multicolumn{4}{c}{ Option } & $\mathbf{4}$ & \multicolumn{4}{c}{ Option } \\
\hline 0 & 0 & 0 & 0 & 0 & 0 & 0 & & 1 & 1 & 1 & 1 & 1 & 1 & 1 \\
0 & 0 & 1 & 1 & 1 & 1 & 0 & 1 & 1 & 0 & 0 & 0 & 0 & 1 \\
0 & 1 & 0 & 1 & 0 & 1 & 1 & & 1 & 0 & 1 & 0 & 1 & 0 & 0 \\
0 & 1 & 1 & 0 & 1 & 0 & 1 & & 1 & 0 & 0 & 1 & 0 & 1 & 0 \\
1 & 0 & 0 & 0 & 1 & 1 & 1 & 0 & 1 & 1 & 1 & 0 & 0 & 0 \\
1 & 0 & 1 & 1 & 0 & 0 & 1 & & 0 & 1 & 0 & 0 & 1 & 1 & 0 \\
1 & 1 & 0 & 1 & 1 & 0 & 0 & & 0 & 0 & 1 & 0 & 0 & 1 & 1 \\
1 & 1 & 1 & 0 & 0 & 1 & 0 & & 0 & 0 & 0 & 1 & 1 & 0 & 1 \\
\hline
\end{tabular}

a 1-1 correspondence between the $\ell_{1}$ levels of the $r^{\text {th }}$ attribute in the first design and the $\ell_{1}$ runs in the second design. We may choose any one of the $\ell_{1}$ level attributes to be replaced.

The constructions presented in this paper allow us to generate designs where $k \leq$ $\left(\ell^{k-z}-1\right) /(\ell-1)$ and $N=\zeta \times \ell^{k-z}$, where $\zeta$ is the number of sets of generators that are used to construct the design. The value for the integer $z$ is chosen to minimise $N$ for a given value of $k$, while ensuring that the strength of the $\mathrm{OA}$ is at least 2 (i.e. the resolution of the design is at least 3). The construction in Burgess and Street (2005) allows us to construct designs where $N=\zeta \times \ell^{k}$. For Example 2.1, given the same set of generators, we would need $N=1 \times 3^{4}=81$ choice sets if we were to use the method in Burgess and Street (2005), rather than the nine choice sets shown in Table 2.2.

We conclude this section with two examples of designs for choice experiments that have been developed from orthogonal arrays.

Example 2.3. Consider the design in Example 2.1. The orthogonal array in the first block of columns of Table 2.2 has been constructed using the Rao-Hamming construction, where the first two columns form the $3^{2}$ complete factorial design, $C_{3}=C_{1}+C_{2}$, and $C_{4}=2 \times C_{1}+C_{2}$, and the addition and multiplication operations are conducted in $G F[3]$.

Example 2.4. Consider a design with $k=7, \ell_{q}=2$ for $1 \leq q \leq 7, m=2$, and $\zeta=1$. To construct the orthogonal array, we begin with the $2^{3}$ complete factorial design, which gives the first three columns of this array. We then add columns of the complete factorial using the following relationships $C_{4}=C_{2}+C_{3}, C_{5}=C_{1}+C_{3}, C_{6}=C_{1}+C_{2}+C_{3}$, and $C_{7}=C_{1}+C_{2}$, where the addition is conducted in $G F[2]$. This gives the first block of columns in Table 2.3. If we add the generator $\boldsymbol{g}_{1,2}=(1111111)$, we obtain the design in Table 2.3.

\section{Optimal Symmetric Designs}

In this section, we construct the information matrix for the estimation of the main effects of the attributes. We then use this to prove a theorem that gives optimal designs for the estimation of main effects for the designs introduced in Section 2. 
In Section 1 of the supplementary material we show that the component of the Fisher information matrix corresponding to the pairs of items in the $i^{\text {th }}$ and $j^{\text {th }}$ positions of the choice sets generated from the $\alpha^{\text {th }}$ set of generators, under the assumption of equal selection probabilities, is given by

$$
C_{\alpha, i, j}\left(\boldsymbol{\pi}_{0}\right)=\frac{1}{m^{2} \zeta \ell^{k}} \operatorname{BlkDiag}\left[B_{\ell}\left(2 I_{\ell}-Q_{g_{\alpha, i, q}-g_{\alpha, j, q}}-Q_{g_{\alpha, j, q}-g_{\alpha, i, q}}\right) B_{\ell}^{T}\right]
$$

where there are $\zeta$ sets of generators used to construct the design, and $Q_{i}$ is an $\ell \times \ell$ permutation matrix where the $(x, y)$ entry equals 1 if $y+i=x$ and 0 otherwise. This matrix consists of $k$ blocks, corresponding to $q=1, \ldots, k$. Using this notation, the Fisher information matrix for the entire experiment, under the assumption of equal selection probabilities, is

$$
C\left(\boldsymbol{\pi}_{0}\right)=\sum_{\alpha=1}^{\zeta} \sum_{i<j} C_{\alpha, i, j}\left(\boldsymbol{\pi}_{0}\right),
$$

since the information matrix for an entire choice set is the sum of the information matrices for each pair of items (Street and Burgess (2007), Section 3.3), and information matrices for separate choice sets are additive.

The expressions in Equations 3.1 and 3.2 can be used to show that a design for a choice experiment is optimal. By observing that the multiset of differences for each attribute $q$ is the set of $g_{\alpha, j, q}-g_{\alpha, i, q}$ values where repeated values are allowed, the next theorem establishes the conditions under which the design will be optimal.

Theorem 3.1. Let $F$ be an orthogonal array obtained from Construction 2.1 with $k$ attributes, each of which have $\ell$ levels. Also let $G_{\alpha}=\left\{\boldsymbol{g}_{\alpha, 1}=\mathbf{0}, \boldsymbol{g}_{\alpha, 2}, \ldots, \boldsymbol{g}_{\alpha, m}\right\}$, for $\alpha=1, \ldots, \zeta$, be a collection of sets of generators such that $\boldsymbol{g}_{\alpha, i} \neq \boldsymbol{g}_{\alpha, j}$ for all $i \neq$ $j$. Suppose that the $i^{\text {th }}$ generator in the $\alpha^{\text {th }}$ set of generators is the $k$-tuple $\boldsymbol{g}_{\alpha, i}=$ $\left(g_{\alpha, i, 1}, g_{\alpha, i, 2}, \ldots, g_{\alpha, i, k}\right)$, for $i=1,2, \ldots, m$. Also suppose that the generators are chosen such that the multiset of differences for each attribute $q$ over all sets in the collection $\uplus_{\alpha=1}^{\zeta} \uplus_{i \neq j}\left(g_{\alpha, j, q}-g_{\alpha, i, q}\right)$ contains each non-zero difference in $G F[\ell]$ equally often. Then the choice sets given by the rows of $F+\boldsymbol{g}_{\alpha, 1}, F+\boldsymbol{g}_{\alpha, 2}, \ldots, F+\boldsymbol{g}_{\alpha, m}$, for $\alpha=1, \ldots, \zeta$, are optimal for the estimation of main effects under the assumption of equal selection probabilities, provided that there are as few zero differences as possible.

A full proof of this theorem is given in Section 2 of the supplementary material. The class of competing designs for this theorem are all designs that can be constructed by adding one or more sets of generators to an orthogonal array constructed using Construction 2.1 where generators satisfy the condition that the multiset of differences for each attribute $q$ over all sets in the collection $\uplus_{\alpha=1}^{\zeta} \uplus_{i \neq j}\left(g_{\alpha, j, q}-g_{\alpha, i, q}\right)$ contains each non-zero difference in $G F[\ell]$ equally often. In the condition of the multiset of differences, we only count the differences for each attribute within each set of generators. In Section 5 we discuss how to determine the minimum number of zero differences for given values of $m, k$, and $\ell$.

If the assumptions that define the class of competing designs hold, then the deter- 
minant of the information matrix for the estimation of main effects is given by

$$
\operatorname{det}\left(C\left(\boldsymbol{\pi}_{0}\right)\right)=\prod_{q=1}^{k}\left(\frac{2 \ell S_{q}}{m^{2} \zeta \ell^{k}(\ell-1)}\right)^{\ell-1}
$$

where there are $2 S_{q}$ non-zero differences in the multiset of differences for attribute $q$. We are particularly interested in non-zero differences between alternatives because it is only when an attribute changes level between alternatives that we obtain information about main effects. Equation 3.3 is equivalent to $\operatorname{det}\left(C\left(\boldsymbol{\pi}_{0}\right)\right)$ in the proof of Theorem 2 of Burgess and Street (2005) when $\ell_{q}=\ell$, for $1 \leq q \leq k$, and $\zeta$ is fixed to equal 1 . Theorem 1 of Burgess and Street (2005) gives an theoretical maximum for the number of non-zero differences for a set of generators for given values of $m$ and $\ell_{q}$.

Theorem 3.2. (Burgess and Street (2005)) The theoretical maximum for the number of non-zero differences for a set of generators for a particular attribute $q$ is

$$
S_{q}^{*}= \begin{cases}\left(m^{2}-1\right) / 4, & \ell_{q}=2, \text { m odd }, \\ m^{2} / 4, & \ell_{q}=2, m \text { even }, \\ \left(m^{2}-\left(\ell_{q} x^{2}+2 x y+y\right)\right) / 2, & 2<\ell_{q}<m, \\ m(m-1) / 2, & \ell_{q} \geq m,\end{cases}
$$

where positive integers $x$ and $y$ satisfy the equation $m=\ell_{q} x+y$ for $0 \leq y<\ell_{q}$.

This theorem assumes that $\zeta=1$. However, since we are only considering the differences within a set of generators, and not differences between generators that belong to different sets of generators, the theoretical maximum for $\zeta$ sets of generators will be $\zeta$ times the theoretical maximum for a single set of generators.

Example 3.1. For the design in Table 2.2 , we have $G_{1}=(0000,1212,2121)$. For the first attribute, the ordered pairs of generator entries $(0,1),(1,2)$, and $(2,0)$ have difference 1 , and the ordered pairs of generator entries $(1,0),(2,1)$, and $(0,2)$ have difference 2 , where the differences are calculated in $G F[3]$. For each attribute, there are three pairs of positions with difference 1 and three pairs of positions with difference 2 , so $S_{q}=3$ for $1 \leq q \leq 4$. By Theorem 3.2, the theoretical maximum number of non-zero differences for $m=3$ is $S_{q}^{*}=3$, for $1 \leq q \leq 4$. Since this design achieves this theoretical maximum, the design in Table 2.2 is optimal by Theorem 3.1 for $m=3$.

We now consider asymmetric designs whose levels are different powers of the same prime.

\section{Optimal Asymmetric Designs via Expansive Replacement}

In this section, we present a theorem that gives optimal designs when the starting design has been constructed using any number of expansive replacements, as defined in Construction 2.2. As above, we assume that all OAs are constructed using the Rao-Hamming method.

In general, we begin by replacing one of the $\ell_{1}$ level attributes in an $O A\left[N, k, \ell_{1}, 2\right]$ with an $O A\left[\ell_{1}, k_{2}, \ell_{2}, 2\right]$. We continue by replacing a different $\ell_{1}$ level attribute with a 
Table 4.1: The orthogonal arrays used in Example 4.1

\begin{tabular}{lllll}
\hline 0 & 0 & 0 & 0 & 0 \\
0 & 1 & 1 & 1 & 1 \\
0 & 2 & 2 & 2 & 2 \\
0 & 3 & 3 & 3 & 3 \\
1 & 0 & 1 & 2 & 3 \\
1 & 1 & 0 & 3 & 2 \\
1 & 2 & 3 & 0 & 1 \\
1 & 3 & 2 & 1 & 0 \\
2 & 0 & 2 & 3 & 1 \\
2 & 1 & 3 & 2 & 0 \\
2 & 2 & 0 & 1 & 3 \\
2 & 3 & 1 & 0 & 2 \\
3 & 0 & 3 & 1 & 2 \\
3 & 1 & 2 & 0 & 3 \\
3 & 2 & 1 & 3 & 0 \\
3 & 3 & 0 & 2 & 1 \\
\hline & & $\mathbf{a}$ & &
\end{tabular}

\begin{tabular}{lll}
\hline 0 & 0 & 0 \\
0 & 1 & 1 \\
1 & 0 & 1 \\
1 & 1 & 0 \\
\hline & &
\end{tabular}

b

\begin{tabular}{lllllll}
\hline 0 & 0 & 0 & 0 & 0 & 0 & 0 \\
0 & 1 & 1 & 1 & 0 & 1 & 1 \\
0 & 2 & 2 & 2 & 1 & 0 & 1 \\
0 & 3 & 3 & 3 & 1 & 1 & 0 \\
1 & 0 & 1 & 2 & 1 & 1 & 0 \\
1 & 1 & 0 & 3 & 1 & 0 & 1 \\
1 & 2 & 3 & 0 & 0 & 1 & 1 \\
1 & 3 & 2 & 1 & 0 & 0 & 0 \\
2 & 0 & 2 & 3 & 0 & 1 & 1 \\
2 & 1 & 3 & 2 & 0 & 0 & 0 \\
2 & 2 & 0 & 1 & 1 & 1 & 0 \\
2 & 3 & 1 & 0 & 1 & 0 & 1 \\
3 & 0 & 3 & 1 & 1 & 0 & 1 \\
3 & 1 & 2 & 0 & 1 & 1 & 0 \\
3 & 2 & 1 & 3 & 0 & 0 & 0 \\
3 & 3 & 0 & 2 & 0 & 1 & 1 \\
\hline
\end{tabular}

$O A\left[\ell_{1}, k_{3}, \ell_{3}, 2\right]$, and so on until the design has the the desired number of attributes with each number of levels. Suppose that after $v$ applications of the expansive replacement algorithm we have $k_{1} \ell_{1}$-level attributes, $k_{2} \ell_{2}$-level attributes, and so on up to $k_{v} \ell_{v^{-}}$ level attributes, where $k_{1}=k-z-v$, and $\ell_{1}, \ell_{2}, \ldots, \ell_{v}$ are all powers of the same prime, and are not necessarily distinct. We label the $\ell_{r}$ level attributes with $r_{1}, \ldots, r_{k_{r}}$.

Example 4.1. In this example, we consider the construction of a DCE design by developing a design constructed using expansive replacement. We begin with two orthogonal arrays of strength two, $O A[16,5,4,2]$, and $O A[4,3,2,2]$, as shown in Table 4.1(a) and Table 4.1(b) respectively. As each entry of the first orthogonal array takes one of four values, and the second orthogonal array has four runs, we may replace the entries in the final column of the $O A[16,5,4,2]$ with the rows of $O A[4,3,2,2]$, where the entry in the final column of the former orthogonal array determines which row of the latter orthogonal array replaces it. In this case, $\ell_{1}=4, \ell_{2}=2, k_{1}=4, k_{2}=3$, and $v=1$. This gives the design in Table 4.1(c). We can then develop this new orthogonal array using the set of generators $G=(0000000,1111111,2222000,3333111)$ to give a choice design. Note that the entries in these generators have been chosen using Table 5.1.

In Section 3 of the supplementary material, we show that if we use generators to develop the type of design described above, then the information matrix for the estimation of main effects is

$$
C_{\alpha, i, j}\left(\boldsymbol{\pi}_{0}\right)=\frac{1}{m^{2} \zeta \ell_{1}^{k}} \operatorname{BlkDiag}\left[B_{\ell_{r}}\left(2 I_{\ell_{r}}-Q_{g_{\alpha, i, r_{q}}-g_{\alpha, j, r_{q}}}-Q_{g_{\alpha, j, r_{q}}-g_{\alpha, i, r_{q}}}\right) B_{\ell_{r}}^{T}\right],
$$

for $r=1, \ldots, v$ and $q=r_{1}, \ldots, r_{k_{r}}$, where $\ell_{1}$ is the number of levels each attribute in the 
original $O A\left[N, k, \ell_{1}, 2\right]$ may take. The first $k_{1}$ blocks are of dimension $\left(\ell_{1}-1\right) \times\left(\ell_{1}-1\right)$, the next $k_{2}$ blocks are of dimension $\left(\ell_{2}-1\right) \times\left(\ell_{2}-1\right)$ and so on.

The following theorem gives optimal designs for the estimation of main effects when the starting design has been constructed using expansive replacement from a RaoHamming design.

Theorem 4.1. Let $F$ be an $O A\left[N ; \ell_{1}^{k_{1}}, \ell_{2}^{k_{2}}, \ldots, \ell_{v}^{k_{v}} ; 2\right]$ that has been constructed by applying Construction 2.2 to $O A\left[N, k, \ell_{1}, 2\right]$ using the orthogonal arrays $O A\left[\ell_{1}, k_{2}, \ell_{2}, 2\right], \ldots$, $O A\left[\ell_{1}, k_{v}, \ell_{v}, 2\right]$, where each orthogonal array has been constructed using Construction 2.1 and $\ell_{1}, \ell_{2}, \ldots, \ell_{v}$ are powers of the same prime. Also let $G_{\alpha}=\left\{\boldsymbol{g}_{\alpha, 1}=\mathbf{0}, \boldsymbol{g}_{\alpha, 2}, \ldots, \boldsymbol{g}_{\alpha, m}\right\}$, for $\alpha=1, \ldots, \zeta$, be a collection of sets of generators such that $\boldsymbol{g}_{\alpha, i} \neq \boldsymbol{g}_{\alpha, j}$ for all $i \neq j$. Suppose that the $i^{\text {th }}$ generator in the $\alpha^{\text {th }}$ set of generators is the $k$-tuple $\boldsymbol{g}_{\alpha, i}=$ $\left(g_{\alpha, i, 1}, g_{\alpha, i, 2}, \ldots, g_{\alpha, i, k}\right)$, for $i=1,2, \ldots, m$. Also suppose that the generators are chosen such that the multiset of differences for each attribute $q$ over all sets in the collection $\uplus_{\alpha=1}^{\zeta} \uplus_{i \neq j}\left(g_{\alpha, j, q}-g_{\alpha, i, q}\right)$ contains each non-zero difference in $G F\left[\ell_{r}\right]$ equally often. Then the choice sets given by the rows of $F+\boldsymbol{g}_{\alpha, 1}, F+\boldsymbol{g}_{\alpha, 2}, \ldots, F+\boldsymbol{g}_{\alpha, m}$, for $\alpha=1, \ldots, \zeta$, are optimal for the estimation of main effects under the assumption of equal selection probabilities, provided that there are as few zero differences as possible.

A full proof of this theorem is given in Section 4 of the supplementary material. By taking the determinant of the Fisher information matrix for the estimation of main effects we obtain

$$
\operatorname{det}\left(C\left(\boldsymbol{\pi}_{0}\right)\right)=\prod_{r=1}^{v} \prod_{q=1}^{k_{r}}\left(\frac{2 \ell_{r} S_{r_{q}}}{m^{2} \zeta\left(\ell_{r}-1\right) \ell_{1}^{k}}\right)^{\ell_{r}-1} .
$$

If we set $\zeta=1$, then this is equivalent to the expression for $\operatorname{det}\left(C\left(\boldsymbol{\pi}_{0}\right)\right)$ in the proof of Theorem 2 of Burgess and Street (2005). Again, we can use Theorem 3.2 to find the theoretical maximum values for $S_{q_{i}}$ for given values of $m, k_{1}, \ldots, k_{v}, \ell_{1}, \ldots, \ell_{v}$.

Example 4.2. For the design described in Example 4.1, we have $\boldsymbol{g}_{2}=(1111111), \boldsymbol{g}_{3}=$ (2222000), and $\boldsymbol{g}_{4}=(3333111)$. So, for $1 \leq q \leq 4, S_{q}=6$ and there are no zero differences. For $5 \leq q \leq 7, S_{q}=4$ and there are four zero differences. Using Theorem 3.2 , we have $S_{1}^{*}=S_{2}^{*}=S_{3}^{*}=S_{4}^{*}=6$ and $S_{5}^{*}=S_{6}^{*}=S_{7}^{*}=4$, confirming that this design has as few zero differences as possible for $m=4$, so this designs is optimal for $m=4$ by Theorem 4.1. It is worth noting that when $\ell<m$ it is not possible to find a set of generators with no zero differences.

\section{Practical Considerations for Constructing Designs}

The designs constructed using the results presented in this paper require both a starting design and a collection of sets of generators. Appropriate starting designs can be found on Neil Sloane's web site, which was given in Section 2. The selection of appropriate sets of generators is more complex, and is discussed in this section.

For Theorems 2.1, 3.1, and 4.1, the goal is to find a collection of sets of generators $G_{1}, G_{2}, \ldots, G_{\zeta}$ such that, for each attribute, each non-zero difference appears equally often in the multiset $\uplus_{\alpha=1}^{\zeta} \uplus_{i \neq j}\left(g_{\alpha, j, q}-g_{\alpha, i, q}\right)$, and the number of zero differences in the multiset is minimised. We only consider the differences between generator entries 
Table 5.1: Sets of generators that satisfy the conditions in Theorem 3.1

\begin{tabular}{|c|c|c|c|c|c|c|c|}
\hline \multirow[b]{2}{*}{$\ell$} & \multicolumn{3}{|c|}{$\mathbf{m}$} & \multirow[b]{2}{*}{$\ell$} & \multicolumn{3}{|c|}{$\mathbf{m}$} \\
\hline & 2 & 3 & 4 & & 2 & 3 & 4 \\
\hline 2 & $(0,1)$ & $(0,1,0)$ & $(0,1,0,1)$ & 8 & $(0,1)$ & $(0,1,3)$ & $(0,1,3,4)$ \\
\hline 3 & $(0,1)$ & $(0,1,2)$ & $(0,1,2,1)$ & & $(0,2)$ & $(0,2,3)$ & $(0,1,4,7)$ \\
\hline \multirow[t]{5}{*}{4} & $(0,1)$ & $(0,1,3)$ & $(0,1,2,3)$ & & $(0,3)$ & $(0,1,4)$ & $(0,1,4,5)$ \\
\hline & $(0,2)$ & & & & $(0,4)$ & $(0,3,4)$ & $(0,2,4,6)$ \\
\hline & $(0,3)$ & & & & $(0,5)$ & $(0,1,2)$ & \\
\hline & & & & & $(0,6)$ & $(0,2,5)$ & \\
\hline & & & & & $(0,7)$ & $(0,2,4)$ & \\
\hline
\end{tabular}

within a particular set of generators $G_{\alpha}$, and not between sets of generators. Street and Burgess (2008) discuss the difficulties in choosing a collection of sets of generators that satisfy the conditions of Theorem 2.1, and conjecture that there may be better ways of using the theorem. These same issues exist for Theorems 3.1 and 4.1.

In Table 5.1, we give generator entries for $\ell_{q}=2,3,4$, and 8 , and $m=2,3$, and 4 . Each set of generator $m$-tuples achieve the theoretical minimum values for $S_{q}^{*}$, as given in Theorem 3.2, while satisfying the conditions of Theorems 2.1, 3.1, and 4.1, that the multiset of differences across all sets of generators, $\uplus_{\alpha=1}^{\zeta} \uplus_{i \neq j}\left(g_{\alpha, j, q}-g_{\alpha, i, q}\right)$, contains each non-zero difference equally often. We demonstrate the use of this table in the following example.

Example 5.1. Consider an experiment with $m=2, \ell_{1}=\ell_{2}=\ell_{3}=4$ and $\ell_{4}=3$. For the four level attributes, Table 5.1 gives three generator pairs, $(0,1),(0,2)$, and $(0,3)$. For the three level attribute, Table 5.1 gives one generator pair, $(0,1)$. The lowest common multiple of the number of generator pairs for the four level attributes and the number of generator pairs for the three level attributes is three, so if we let $\zeta=3$ we can obtain a collection of sets of generators where, for each attribute, the multiset of differences across all sets of generators will contain each non-zero difference equally often. We use the $(0,1)$ pair in each set of generators for the three level attribute. So $G_{1}=\{(0000),(1111)\}, G_{2}=\{(0000),(2221)\}$, and $G_{3}=\{(0000),(3331)\}$.

We may decide to reorder the generator entries for a particular attribute within a set of generators, perhaps to avoid dominated alternatives, that is, where one alternative has 'more desirable' levels than another alternative for every attribute. If we reorder the generator entries for the first attribute in $G_{1}$ and $G_{3}$, but not $G_{2}$, we have $G_{1}=$ $\{(1000),(0111)\}, G_{2}=\{(0000),(2221)\}$, and $G_{3}=\{(3000),(0331)\}$, which still satisfy the conditions of Theorems 2.1, 3.1, and 4.1.

We may also change the assignment of the generator pairs to $G_{1}, G_{2}$, and $G_{3}$ for a particular attribute. For instance we could assign $(0,2)$ to $G_{1},(0,1)$ to $G_{2}$, and $(0,3)$ to $G_{3}$ for the first attribute to obtain $G_{1}=\left\{(0000),(2111), G_{2}=\{(0000),(1221)\}\right.$, and $G_{3}=\{(0000),(3331)\}$, which still satisfies the conditions for Theorems 2.1, 3.1, and 4.1 .

In the previous example, we described two approaches to reordering generator en- 
tries. The purpose of the reordering is not to improve the efficiencies of the design, as the designs are equally efficient. The idea is to be able to move between optimal designs so that other criteria may be satisfied as well. One common criterion is the avoidance of dominated alternatives.

Street and Burgess (2007) provide a tool for checking the efficiency of designs using the method described in this paper. This tool can be found at http://maths. science. uts.edu.au/maths/wiki/SPExpts. To use the software, values for $m, k$, and $\ell_{q}$ for $q=1,2, \ldots, k$ must be given, and users need to specify $F$ and $G_{\alpha}$ for $\alpha=1,2, \ldots, \zeta$. The tool will then return the $D$-efficiency of the design, as well as the $B, \Lambda\left(\boldsymbol{\pi}_{0}\right), C\left(\boldsymbol{\pi}_{0}\right)$, and $\left(C\left(\boldsymbol{\pi}_{0}\right)\right)^{-1}$ matrices. This software assumes that $\boldsymbol{g}_{\alpha, 1}=\mathbf{0}$ for $\alpha=1,2, \ldots, \zeta$.

\section{Conclusion}

We conclude by providing a comparison of the number of choice sets required for various construction methods of optimal designs. In particular, we compare the construction method discussed in this paper to the constructions presented in Burgess and Street (2005) and Graßhoff et al. (2004), which both focus on main effects designs. This comparison is shown for symmetric designs with $m=2$ in Table 6.1. In addition to these, Graßhoff et al. (2004) gives a construction for $\ell=3$ which requires either $3 k$ or $9 k / 4$ choice sets based on Latin and Graeco-Latin squares (p369), and for $k=2$ which requires $\ell(\ell-1) / 2$ choice sets based on Bhaskar-Rao designs (p370). The authors mention that the construction based on Latin and Graeco-Latin squares can be extended for $\ell>3$. For $m=2$, the methods given in Graßhoff et al. (2004) give the fewest choice sets, but do not give designs for $m>2$. The methods presented in Burgess and Street (2005) and in this paper allow for the construction of comparatively small designs when $m>2$. For instance, when $\ell=4$, we can reduce the number of choice sets required by two thirds by letting $m=3$ or $m=4$.

Unlike Burgess and Street (2005), the designs that are considered in this paper are quite restrictive in the number of levels each attribute may take. The design optimality results only apply to symmetric experiments with a prime power number of levels, and some cases where the attributes may take different numbers of levels, all of which are powers of the same prime. It is possible to construct fractional factorial designs that have attributes with a number of levels that is not a prime power, or that are asymmetric but constructed using methods other than those covered here. Some of these constructions are given in Chapters 9 and 10 of Hedayat et al. (1999). Future research needs to be undertaken to adapt the methods used here to extend the design optimality results to designs that are developed from orthogonal arrays constructed using other methods. Future research also needs to be conducted to provide a general result that gives the optimal set of generators required in Theorems 2.1, 3.1, and 4.1.

\section{Acknowledgements}

The author would like to thank Professor Deborah Street for providing support and sound advice through the research and review phases of this paper. The author would also like to thank the referees for their constructive comments on this article, which have substantially improved the clarity of the presentation of this article. 
Table 6.1: Required number of choice sets for $m=2$

\begin{tabular}{rrrrrr}
\hline $\mathbf{k}$ & $\boldsymbol{\ell}$ & $\begin{array}{c}\text { Proposed } \\
\text { Construction }\end{array}$ & $\begin{array}{c}\text { Burgess and } \\
\text { Street (2005) }\end{array}$ & $\begin{array}{c}\text { Graßhoff et al. } \\
\text { p366 }\end{array}$ & $\begin{array}{c}\text { (2004) } \\
\text { p368 }\end{array}$ \\
\hline 2 & 2 & 4 & 4 & 2 & 4 \\
& 3 & 9 & 9 & 6 & 9 \\
& 4 & 48 & 48 & 12 & 36 \\
3 & 2 & 4 & 8 & 4 & 4 \\
& 3 & 9 & 27 & 12 & 9 \\
& 4 & 48 & 192 & 24 & 36 \\
4 & 2 & 8 & 16 & 4 & 8 \\
& 3 & 9 & 81 & 12 & 9 \\
& 4 & 48 & 768 & 24 & 36 \\
5 & 2 & 8 & 32 & 8 & 8 \\
& 3 & 27 & 243 & 24 & 27 \\
& 4 & 48 & 3072 & 48 & 36 \\
6 & 2 & 8 & 64 & 8 & 8 \\
& 3 & 27 & 729 & 24 & 27 \\
& 4 & 192 & 12288 & 48 & 216 \\
7 & 2 & 8 & 128 & 8 & 8 \\
& 3 & 27 & 2187 & 24 & 27 \\
& 4 & 192 & 49152 & 48 & 216 \\
8 & 2 & 16 & 256 & 8 & 16 \\
& 3 & 27 & 6561 & 24 & 27 \\
& 4 & 192 & 196608 & 48 & 216 \\
\hline
\end{tabular}




\section{References}

Bradley, R.A., Terry, M.E., 1952. Rank analysis of incomplete block designs: I. The method of paired comparisons. Biometrika, 39, 324-345.

Burgess, L., Street, D.J., 2003. Optimal designs for $2^{k}$ choice experiments. Communications in Statistics - Theory and Methods, 32, 2185-2206.

Burgess, L., Street, D.J., 2005. Optimal designs for choice experiments with asymmetric attributes. Journal of Statistical Planning and Inference, 134, 288-301.

Burgess, L., Street, D.J., Wasi, N., 2011. Comparing designs for choice experiments: A case study. Journal of Statistical Theory and Practice, 5, 25-46.

Carson, R.T., Louviere, J.J., Wei, E., 2009. Alternative Australian climate change plans: The public's views. Energy Policy, 38, 902-911.

El-Helbawy, A.T., Bradley, R.A., 1978. Treatment contrasts in paired comparisons: Large-sample results, applications, and some optimal designs. Journal of the American Statistical Association, 73, 831-839.

Graßhoff, U., Großmann, H., Holling, H., Schwabe, R., 2004. Optimal designs for main effects in linear paired comparison models. Journal of Statistical Planning and Inference, $126,361-376$.

Graßhoff, U., Schwabe, R., 2008. Optimal design for the Bradley-Terry paired comparison model. Statistical Methods and Applications, 17, 275-289.

Großmann, H., Holling, H., Graßhoff, U., Schwabe, R., 2007. A comparison of efficient designs for choices between two options. MODA 8-Advances in Model-Oriented Design and Analysis, Physica-Verlag, Heidelberg, 83-90.

Hamming, R.W., 1950. Error-detecting and error correcting codes. Bell System technical journal, 29, 147-160.

Hedayat, A.S., Sloane, N.J.A., Stufken, J., 1999. Orthogonal arrays: Theory and applications, Springer-Verlag, New York NY.

Jaeger, S.R., Rose, J.M., 2008. Stated choice experimentation, contextual influences and food choice: A case study. Food Quality and Preference, 19, 539-564.

Kessels, R., Jones, B., Goos, P., Vandebroek, M., 2009. An efficient algorithm for constructing Bayesian optimal choice designs. Journal of Business and Economic Statistics, 27, 279-291.

Lancsar, E.J., Hall, J.P., King, M., Kenny, P., Louviere, J.J., Fiebig, D.G., Hossain, I., Thien, F.C.K., Reddel, H.K., Jenkins, C.R., 2007. Using discrete choice experiments to investigate subject preferences for preventive asthma medication. Respirology, 12, 127-136.

Louviere, J.J., Hensher, D.A., Swait J.D., 2000. Stated choice methods, Cambridge University Press, Cambridge. 
Rao, C.R., 1947. Factorial experiments derivable from combinatorial arrangements of arrays. Supplement to the Journal of the Royal Statistical Society, 9, 128-139.

Rao, C.R., 1949. On a class of arrangements. Proceedings of the Edinburugh Mathematical Society, 8, 119-125.

Street, D.J., Burgess, L., 2004. Optimal and near-optimal pairs for the estimation of effects in 2-level choice experiments. Journal of Statistical Planning and Inference, 118, 185-199.

Street, D.J., Burgess, L., 2007. The Construction of optimal stated choice experiments: Theory and methods, Wiley, Hoboken NJ.

Street, D.J., Burgess, L., Louviere, J.J., 2005. Quick and easy choice sets: Constructing optimal and nearly optimal stated choice experiments. International Journal of Research in Marketing, 22, 459-470.

Street, D.J., Burgess, L., 2008. Some open combinatorial problems in the design of stated choice experiments. Discrete Mathematics, 308, 2781-2788.

Train, K.E., 2003. Discrete choice methods with simulation, Cambridge University Press, New York NY. 